\title{
3D MODELLING AND VIRTUAL REALITY APPLIED TO COMPLEX ARCHITECTURES: AN APPLICATION TO HOSPITALS' DESIGN
}

\author{
P. Chias ${ }^{1}$, T. Abad ${ }^{1}$, M. de Miguel $^{1}$, G. García-Rosales ${ }^{1}$, E. Echeverria ${ }^{1}$ \\ ${ }^{1}$ School of Architecture, University of Alcalá, Madrid, Spain, (pilar.chias, tomas.abad, manuel.miguel, g.rosales, \\ ernesto.echeverria)@uah.es
}

\section{Commission II}

KEY WORDS: HBIM, healthcare facilities, 3D modelling, hospitals' management, laser scanning

\begin{abstract}
:
There are many healthcare facilities in Spain that were built throughout the second half of the $20^{\text {th }}$ century, and compose an interesting built heritage designed by outstanding architects of the architectural avant-garde. Some of them were included in the DOCOMOMO's list of buildings to be preserved and documented. As a consequence, every intervention on the hospitals' heritage requires a special attention and needs a deep knowledge of the building in advance of taking action. On the other hand, such a particular heritage is being continuously updated according to the new standards that derive from the current tendencies in hospital design, and also from advances in healing techniques. In this context, 3D Modelling and virtual reality should have a decisive impact on the way hospitals' complex spaces are designed, as they become useful tools for describing the previous stages of the buildings, as well as the impacts of new proposals. They can even change our experience of the built environment. As a step forward, we are developing a methodology to be applied to hospital design where these tools, together with BIM models -and in the near future, high-end immersive VR systems- play an important role.
\end{abstract}

\section{INTRODUCTION}

Healthcare facilities become a particularly interesting built heritage when they are designed by outstanding architects. This is the case of some projects developed throughout the $20^{\text {th }}$ century by some representatives of the Spanish avant-garde as Fernando García Mercadal and Rafael Sánchez Arcas, among other (Figure 1).

Their works were included in the DOCOMOMO's list of buildings to be preserved and documented, and as a consequence, each intervention on such a particular heritage requires a special attention, and needs a deep knowledge of the building in advance of taking action.

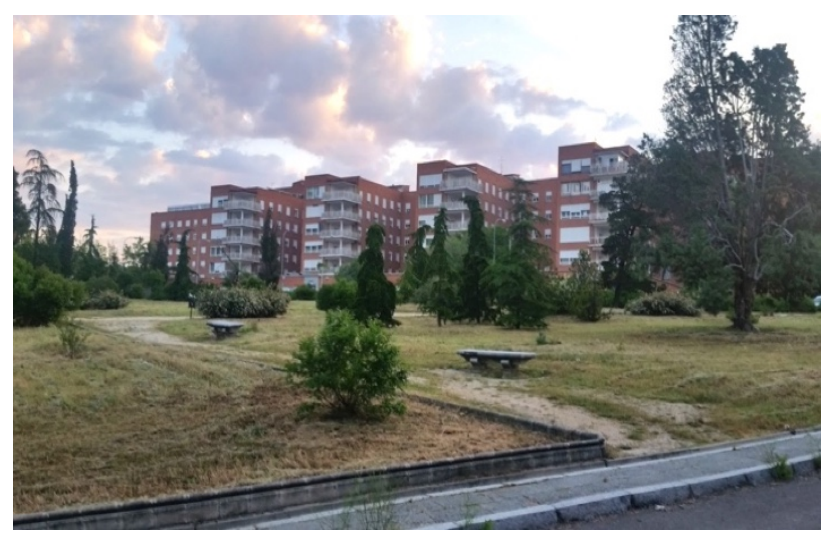

Figure 1. Rafael Sánchez Arcas 1933-1936, Hospital Clínico San Carlos, Madrid.

Such a built heritage must be continuously updated due to the increasing need to upgrade the existing healthcare facilities, and to make them fit with the new standards deriving from current tendencies in hospital design, and also from advances in healing techniques.

Concepts as "feel at home", "healing gardens", "e-Health" or "green hospitals" are being applied progressively in the old buildings, and their consequences should be foreseen in advance and reconciled in a forward-looking respectful strategy.

In this context, 3D Modelling, BIM and virtual reality should have a decisive impact on the way the hospitals' complex spaces are designed, because they become useful tools for describing the previous stages of the buildings, as well as to forecast the impacts of the new proposals. They can even change our experience of the built environment.

Our main innovative target is to develop an integrative BIMbased methodology that serves as a document manager for the collection and management of the whole information system of the hospital, as well as for the coordination of both the design and the construction stages.

As a secondary target, the 3D model will be useful to propose diferent design possibilities for indoor spaces, and to check the users' reactions in order to achieve the best solution in terms of comfort and wellbeing.

\subsection{Case study}

The University Hospital Príncipe de Asturias is located in the University Campus of Alcalá de Henares, $5 \mathrm{~km}$ far from the city centre and well communicated by public transport, by rail and road, both with Madrid and Guadalajara (Figure 2).

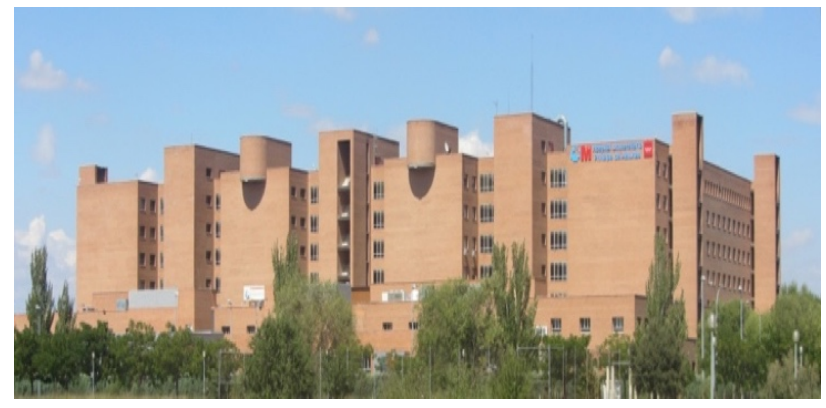

Figure 2. The University Hospital Príncipe de Asturias, Alcalá de Henares, Madrid. 
From the typological point of view, the hospital is an isolated flat roofed eight storey building, consisting of four wings linked by two long corridors running parallel from NW to SE.

A small building was added in 2015 in the northwestern area of the hospital's plot, to be used for training purposes and cafeteria. A new Emergency Department was also built a couple of years ago, that was located inside a new two storey building adjacent to the old one by the southwestern façade.

Service buildings and other facilities as the power plant, the medicinal gas plant, the central $\mathrm{A} / \mathrm{C}$ unit, and the hydraulic center, are located in a separate construction that is located southeast of the main building.

The hospital is an interesting highly complex healthcare facility built in 1993 (Table 1).

\begin{tabular}{lllll}
\hline Year & $\begin{array}{l}\text { Nr. of } \\
\text { beds }\end{array}$ & $\begin{array}{l}\text { Site } \\
\text { surface } \\
\mathbf{m}^{2}\end{array}$ & $\begin{array}{l}\text { Built } \\
\text { surface } \\
\mathbf{m}^{2}\end{array}$ & Typology \\
\hline 1993 & 594 & 84.000 & 81.930 & 4 wings, 8 \\
2015 & & & 93.000 & $\begin{array}{l}\text { storeys, and 2 } \\
\text { enlargements }\end{array}$ \\
\hline
\end{tabular}

Table 1. General specifications for the University Hospital Príncipe de Asturias.

The complex is surrounded by a garden that is currently crossed by access roads that are used as parking areas, with a total of 600 ground parking places (Figures 2 and 3 ).

Their high complex organisation derives, on the one hand, from their particular technical, functional, and instrumental constraints. And on the other hand, from the specific problems set by the needs of their complex spatial relationships.

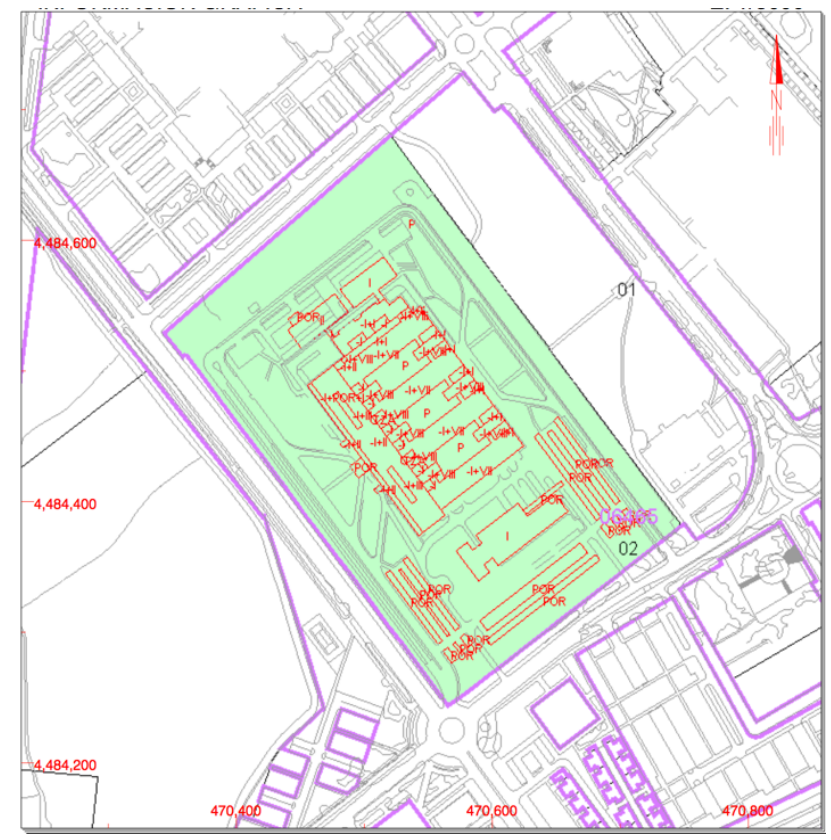

Figure 3. Cadastral map of the University Hospital Príncipe de Asturias.

\section{PRECEDENTS AND RELATED WORKS}

One of the main challenges launched by the European Commission within the Societal Challenge Work Program of HORIZON 2020 concerns the management of accurate low cost Modelling with Revit is a fast solution for new constructions.
It is due, on the one hand, to the advantages of importing point clouds by Recap, and to the direct connectivity with the scan station Leica. The Cloudworks plug-in available in AutoCAD is also a useful way to draw 2D maps and plans, and 3D perspectives, from the point cloud datasets.

And on the other hand, because it offers a simple quick method for modelling, as well as an easy access to digital models.

There are various recent precedents on 3D Modelling and virtual reality researches applied to various types of cultural heritage buildings.

Adami, Scala and Spezzoni (2017) discussed the difficulties and potentialities of combining an approach based on standardization with the historical reality, which is made of unique elements (Benatti, Borgarino, and Della Torre, 2014).

Though there are no precedents that focuse particularly on healthcare facilities, some interesting approaches deal with the study of complex architectures and sites (Di Giulio, et al., 2017) by integrating the data acquisition technologies and management, and diagnostic skills on an HBIM environment.

Relevant improvements in the performance and practices that give advantage in terms of accuracy, acquisition and processing times, and costs have been widely discussed by Remondino and Campana (2014), Guidi et al. (2014) and Fassi et al. (2013), among other.

Many studies focused on the potential of the laser scanner techniques in the architectural survey field (Rinaudo, et al., 2007; Andrews, et al., 2010; Cardaci, et al., 2013; De Luca, et al., 2016).

By means of some scan to BIM strategies, the parametric model brings the point cloud scanned elements back to objects to which construction data can be associated, and a chronological mapping can be produced in order to identify in situ traces and transformations carried out over time (Di Luggo, and Catuogno, 2016; Di Luggo, and Scandurra, 2016).

Another challenge deals with cualitative data collection and management, due to the importance of users' experience in hospital construction, update and adaptation to new uses and strategies. About this subject some precedents are from other scientific fields as psychosocial studies, and neuroscience in use for testing users' preferences (Post Sennehed, et al., 2017).

Finally, 3D Models and virtual reality become useful tools to check reactions and of in-patients and accompanying persons (Hsu, Chuang, and Chang, 2000; Castilla, Llinares, and Blanca, 2015).

\section{DEVELOPED METHODOLOGY}

The integrative methodology aims to define a frame regarding both quantitative and qualitative data in an HBIM environment. According to the different targets and strategies, we developed the following phases.

\subsection{Modelling methods and criteria}

BIM modelling represents architecture organised for technological elements, not just for architectural ones. Consequently, the system supports the procedures for managing a conservation and renovation planning.

As we noticed before, modern healthcare facilities are quite standardized, what simplifies the modelling process and reaches a good compromise between the BIM-oriented model and the acquired survey datasets (Figure 4).

As a first step we defined how to break conceptually into small pieces the whole building, and we found out that the best criterion to be followed was to separate the public and private areas, as well as those occupied by services and infrastructures.

Then, all areas were cathegorized in a hierarchical classification taking into account healthcare services and units, technological 
elements, architectural elements, their position, and a sequential numbering.

We also used shared parameters to qualify the various elements, as they are the only kind of parameters that can be included in the schedules, and are useful to manage the model. The schedule of elements is just a list of all objects of the same family, and can be customized in order to show and modify some of their relevant parameters (Adami, Scala, and Spezzoni, 2017).

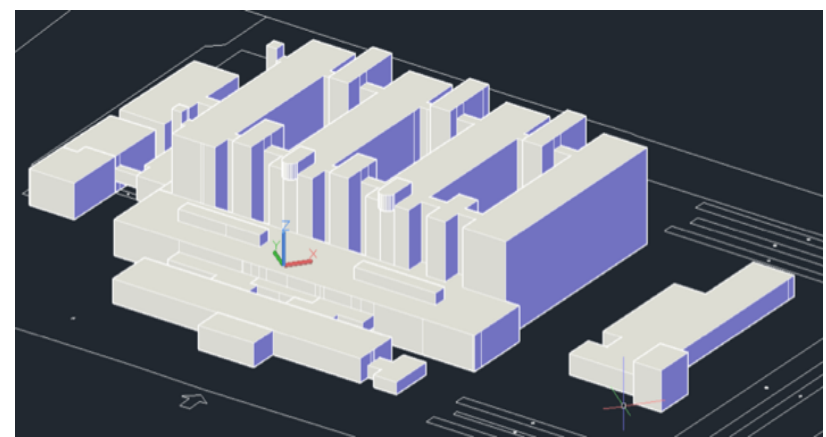

Figure 4. 3D model, Hospital Príncipe de Asturias, Alcalá de Henares.

Among the greatest difficulties encountered during the modelling process, the following can be stressed:

1 / Some were related to the entering of datasets in the BIM databases from various collecting procedures and fieldwork campaigns (Remondino and Campana, 2014), as are the scanning surveys, questionnaires, etc.

2/ The definition of the topological relationships and their validation became particularly complicated, due to the complexity of inside and outside navigation, and to the particular needs of space distribution related to the various shared and un-shared uses of rooms.

\subsection{Survey campaigns and data collection}

The surveying campaing was accomplished in 2018, and lasted a month. Rooms and corridors were surveyed by means of a laser scanner Leica BLK360, and the successive scans were georeferenced in a particular internal reference system that was also used for the 3D model.

High definition images were simultaneously taken by the scan station, and subsequently used for mapping and texturing (Figure $5)$.

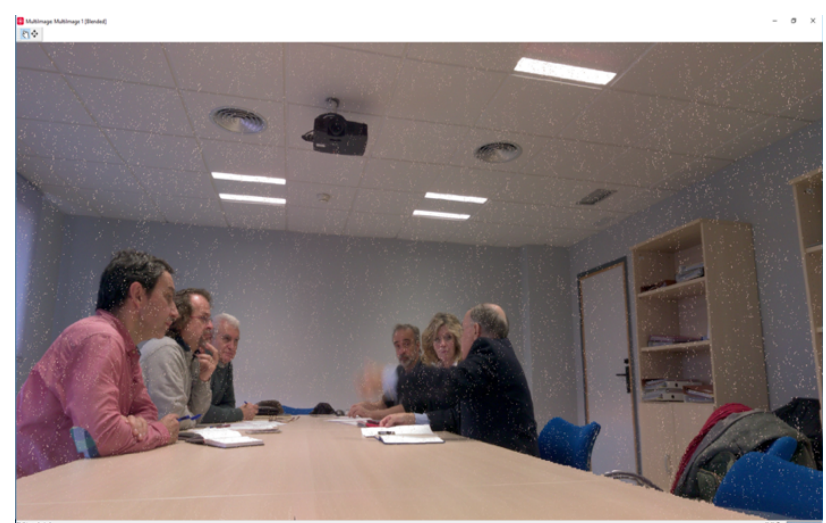

Figure 5. HDR image of a hospital's meeting room, captured by the Leica BLK360 scanner.

Rooms and areas having many technical devices inside, used to need more scans, while public areas and corridors were acquired with two or three scans.
Due to the regularity of shapes and architectural elements, the resolution was set for a medium scan density that provided a 4 mm accuracy.

Walls, floors and ceilings -slabs- are flat surfaces that were easily created in Revit and defined by base constraint, height, constant thickness, together with a layer structure.

Doors, windows and voids were also standardized, and we just created the corresponding wall-based families according to their shapes; their parametric profiles were nested inside the corresponding groups (Figure 6).

All these subsystems have their own methodological approach that run in parallel to the main methodological process.

On the other hand, the possibility of using the AutoCAD Cloudworks plug-in by Leica to draw cross sections was only used in order to produce some overall documents of the hospital.

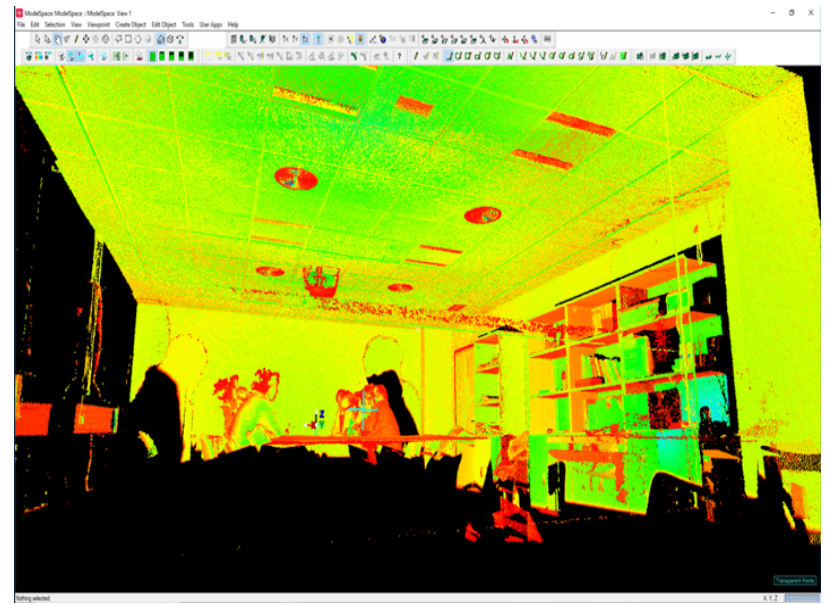

Figure 6. Point cloud of the meeting room.

As the case study was built thirty years ago and has been successively updated, during the data collection and surveying processes we dealt with some particular problems:

$1 /$ as the original facilities use to be non-accesible and hidden behind the walls, some invasive and non-invasive surveying methods were developed.

2/ particular Units as Diagnostic Imaging are protected against radiation hazards by thick lead walls, resulting some additional difficulties in connecting the scanner with the PC or tablet, as well as appearing shadow zones. All these circumstances lead to subsequent problems in the allignment processes.

3/ the intensive use of the building, 24 hours a day and 7 days a week, required surveying campaigns during late night time hours.

4/ short clinical questionnaires were proposed to manage subjective data (Post et al., 2017), involving data protection and privacy. Consequently, the corresponding protocols must be followed.

\subsection{Data organization}

According to the criterion of breaking down the whole building into single elements and areas, the structure of the model reflects this subdivision, that in turn keeps the general categorization in layers as enclosures, facilities, structures, furniture, etc. (Figures 7, 8 and 9).

As the BIM environment can be considered a true 3D GIS at an architectural scale, it exceeds the possibilities of any CAD software labelling process, while it meets the needs of managers but also of biomechanical engineers.

Every facility was easily integrated into the BIM structure as different network systems, where only the oldest ones presented 
some particular challenges in terms of location and tracking (Figure 7).

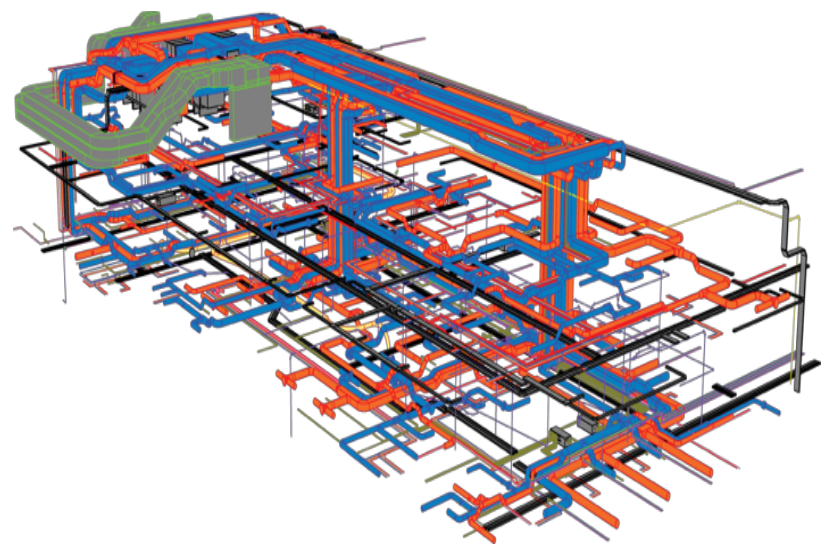

Figure 7. Pipelines as represented in the BIM model.

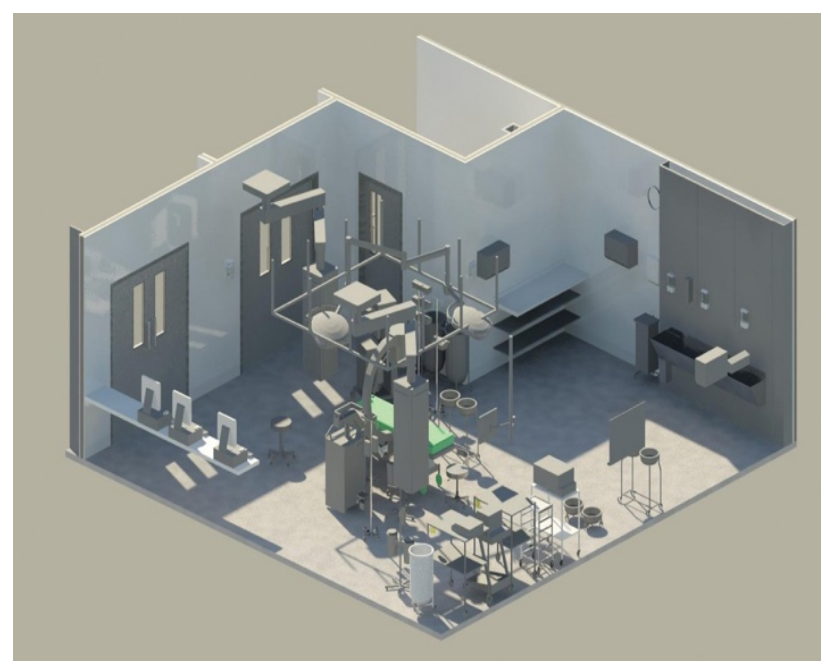

Figure 8 . One of the subdivided areas showing some layers of the BIM.

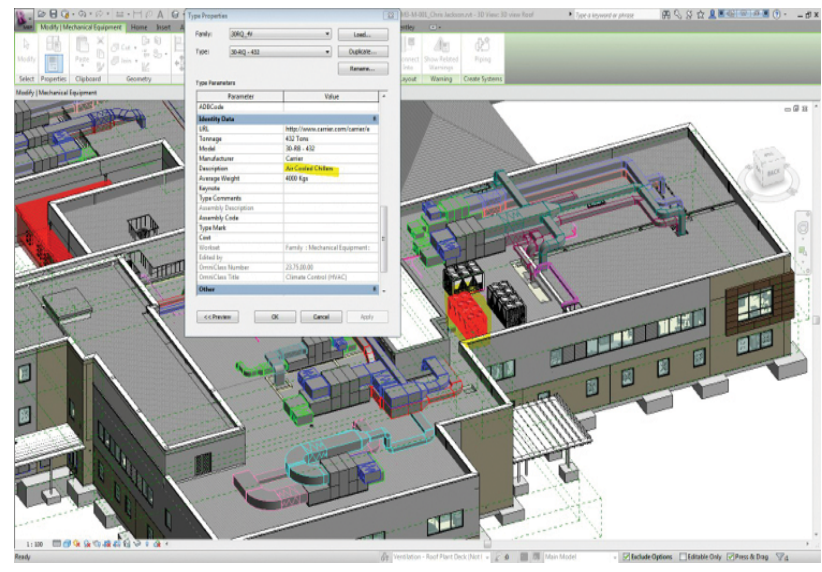

Figure 9. Schedule and view of the model.

Likewise of a GIS graphic basis -map or plan-, topology must be defined between areas and elements in order to permit the adequate queries and to extract and share information between various operators.
Revit enables to extract automatically a schedule applying the same subdivision of areas used in the 3D model, and establishes a direct link between model and schedules. Thus, information of materials, conservation, etc. of an element can be added to the corresponding record, or just by filling in its property window. As a consequence, the system allows to act more directly and easily on any problems which may arise.

The highly standardized constructive solutions adopted for the hospital's design and construction, have greatly facilitated and simplified this phase.

\subsection{D Model to assess users' experience of spaces}

Traditionally, modelling in architecture allows to study a building in all its metric and materic issues, as well as in its spatial organisation. But nowadays they can permit to study not only its quantitative characteristics, but also the qualitative ones. This phase consists of using the 3D model -and in a near future some immersive reality devices- to get the necessary cualitative datasets about spaces and surface finishes.

It has been proved that working conditions and healing environments substantially influence work ability and sick leave. To this end, some members of the research team that are experts on questionnaires, adapted 'The General Nordic Questionnaire' $\left(\right.$ QPS $\left._{\text {Nordic }}\right)$ focusing on work-related psychosocial risk factors and on any potential need for actions at each workplace or area (Post Sennehed et al., 2017).

The research group identified five content areas with a total of 51 items which were considered to be most relevant according to our targets. Fourteen items were then selected from the content areas and organised on a short 'Blue Flags' questionnaire -screening for different health status or risks are often described as different type of clinical 'flags'.

The flag system has been developed for the assessment of risk factors and recommended as a research methodology, as the identification of each coloured flag is established and provides valuable information to clinitians in health care.

Blue flags, for instance, are defined as the individuals' perception of work-related factors that can have an impact on disability. We extended this concept to wider environment-related factors. Results were quantified according to a scale from -3 to +3 points (Table 2).

\begin{tabular}{c|c|c|c|c|c|c|c|c} 
& $\mathbf{3}$ & $\mathbf{2}$ & $\mathbf{1}$ & $\mathbf{0}$ & $\mathbf{- 1}$ & $\mathbf{- 2}$ & $\mathbf{- 3}$ & \\
\hline Pleasant & & & & & & & & $\begin{array}{c}\text { Unpleasa } \\
\text { nt }\end{array}$ \\
\hline High quality & & & & & & & & $\begin{array}{c}\text { Low } \\
\text { quality }\end{array}$ \\
\hline Harmonious & & & & & & & & $\begin{array}{c}\text { Not } \\
\text { harmonic }\end{array}$ \\
\hline Well finished & & & & & & & & $\begin{array}{c}\text { Poorly } \\
\text { finished }\end{array}$ \\
\hline Nice & & & & & & & & Ugly \\
\hline Good & & & & & & & & Bad \\
\hline Complicated & & & & & & & & Simple \\
\hline Common & & & & & & & & Designed \\
\hline Discreet & & & & & & & & Fussy \\
\hline Light & & & & & & & & Heavy \\
\hline Bright & & & & & & & & Dark \\
\hline Original & & & & & & & & Vulgar \\
\hline Happy & & & & & & & & Sad \\
\hline Dark & & & & & & & & Bright \\
\hline
\end{tabular}

Table 2. Semantics and antonyms used to study wall colours and finishes. 
Enquiry was applied this time to a group of in-patients in the Maternity Unit, and results regarded the overall 'Blue flags' to be relevant, with a Content Validity Index (CVI) of $90 \%$.

Consequently, semantic spaces should be included in the HBIM environment. With this purpose, a specific protocol must be designed to measure and assess the users' perceptions and experiences according to a set of variables (Figure 10).

Furthermore, although the application of high-end immersive VR systems is still in progress, our methodology establishes a framework in order to quantify the existing relationships between the environmental design and the users' emotional response.

They allow for an accurate definition of the perceived features as the structure of concepts applied by users to describe and appreciate an indoor environment.
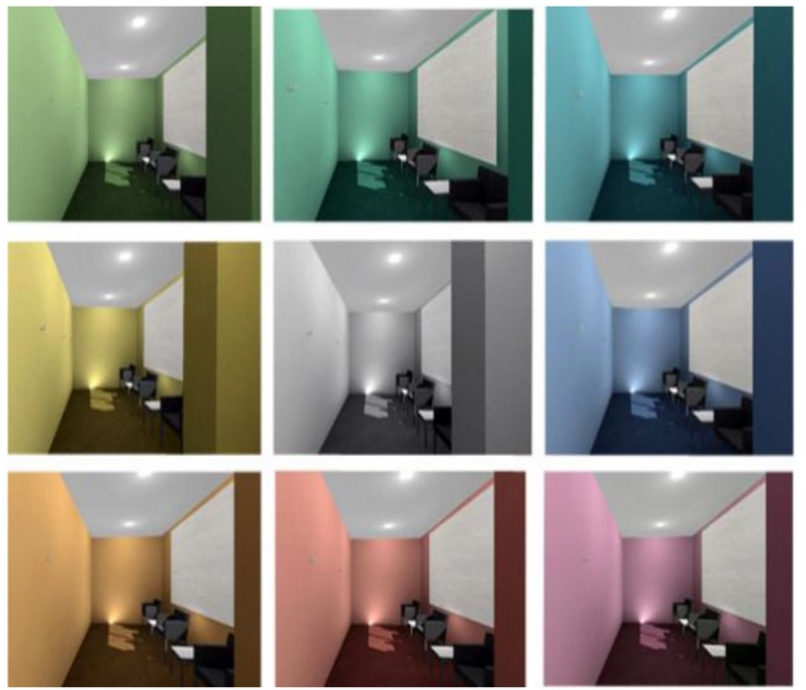

Figure 10. 3D model and virtual reality applied to a lactation room in the Maternity Unit at the Hospital Príncipe de Asturias, Alcalá de Henares.

\subsection{Process validation}

The reliability of the methodology was tested at the end of the process.

Furthermore, the range of admitted errors depends on the characteristics of the architecture, the quality of data, and the purpose of the BIM.

In this regard, it can be used as a 3D index for management and conseervation, for assessing the quality of spaces, or for an architectural intervention or updating.

In addition, the subdivision in small elements or areas of the main architecture was adequately connected with the BIM targets, as it took into account the structural and architectural elements paying a special attention to windows, lighning devices, etc.-, as well as facilities, furniture and finishes, among other.

As we said, the use of the same coordinate system of the laser scanner datasets to build the model, implied that there was no lack of positional accuracy.

On the other hand, modern architecture is highly standardized, and errors due to different thickness were minimized. This makes a significant difference to HBIM applied in the restoration field.

\section{CONCLUSIONS}

The creation of the 3D model in an HBIM environment enriches the interdisciplinary knowledge of complex buildings as hospitals, giving meaning to shapes and geometries thanks to the required ontological identification of each element and their connections by means of a semantic structure.

But while quantitative evaluation criteria such as energy or structural performances are issues usually considered through a building refurbishment process, more qualitative aspects as human satisfaction, staff productivity or patient's comfort and well being are usually neglected and left to the designers' intuition or professional experience.

As an interesting innovation, our methodology integrates both quantitative and qualitative datasets in our BIM model, providing a framework that permits to quantify and assess the users' emotional responses to particular design features.

As a step forward in this subject, our methodology becomes also useful:

- To describe the former or previous stages of the building, its original spaces, characteristics and qualities, before acting. The traces of interventions, structural modifications and changes in the existing facilities are surveyed combining different techniques and recorded in the corresponding databases that will be linked to the BIM model at a later stage. To that end, representations must be based on a systematic reading of the whole building, "in order to document its composition, constructive historical and material characteristics" (Di Luggo and Scandurra, 2016).

- Throughout the design process, 3D models and virtual reality are successfully used by the multidisciplinary design team to assess the impact of each proposed change. They provide a pre-design evaluation before building. But they also facilitate dialogue and interaction with users and stakeholders, and help to encourage feedback and suggestions in order to improve the hospital's design.

With respect to this issue, the modelling tools provide controlled settings in which design hypotheses can be tested before design is finalised. Thus, simulated spaces show new efficiently lit spaces, together with coloured and textured atmospheres that can be easily understood and experienced by the clinical staff as well as by patients and other users.

In a future phase we will explore the way to use the model to know how to get a direct experience of the hospital's spaces that can be enhanced through specialist hardware such as headmounted or large wall-mounted displays and virtual Kansei Engineering techniques.

This experience is currently being applied to assess the emotional response of users with respect to some particular aspects of architectural design such as lighting, colours, and textures.

In short, our research aims to investigate how computational simulation can help not only in hospitals' management, but to document actions taken on their original spaces, and to assess various design proposals as far as they are experienced by people. By simulating various sceneries and patterns with different degrees of complexity, the project team, the clinical staff and the users can ask new types of questions related to the interactions between people and the physical settings.

\section{ACKNOWLEDGEMENTS}

This paper is a result of the research project BIA2016-78893-C3$1-\mathrm{R}$ "Methodology to evaluate comfort requirements, environmental conditions and spatial functionality in hospitals and their surroundings. Proposals to adequate them to the new healthcare concepts".

The authors would like to acknowledge the Spanish Agency of Research of the Spanish Ministry of Economy for its support. We are also grateful to Dr. Diego Rodríguez Puyol, Director of the Foundation Hospital Príncipe de Asturias for his help throughout the research process. 


\section{REFERENCES}

Adami, A., Scala, B., and Spezzoni, A., 2017. Modelling and accuracy in a BIM environment for planned conservation: the Apartment of Troia of Giulio Romano. The International Archives of Photogrammetry, Remote Sensing and Spatial Information Sciences, Vol. XLII-2/W3, doi.org/10.5194/isprsarchives-XLII-2-W3-17-2017.

Andrews, D., Bedford, J., Blake, B., Bryan, P., Cromwell, T., and Lea, R., 2010. Measured and Drawn: Techniques and practice for the metric survey of historic buildings. Historic England, Swindon, UK.

Benatti, E., Borgarino, P., and Della Torre, S., 2014. PlaNet Beni Architettonici. Uno strumento per la conservazione programmata del patrimonio storico-architettonico. In S. Della Torre and P. Borgarino (ed.), ICT per il miglioramento del processo conservativo. Nardini Editore, Firenze, pp. 13-30.

Campi, M., Di Luggo, A., and Scandurra, S., 2017. 3D Modelling for the knowledge of architectural heritage and virtual reconstruction of its historical memory. The International Archives of Photogrammetry, Remote Sensing and Spatial Information Sciences, Vol. XLII-2/W3, doi.org/10.5194/isprsarchives-XLII-2-W3-133-2017.

Cardaci, A., and Versaci, A., 2013. Image-based 3D modelling vs. laser scanning for the analysis of medieval architecture: the case of St. Croce in Bergam. The International Archives of Photogrammetry, Remote Sensing and Spatial Information Sciences, Vol. XL-5/W2, doi.org/10.5194/isprs-archives-XL-5W2-155-2013.

Castilla, N., Llinares, C., and Blanca, V., 2015. Ingeniería Kansei aplicada al diseño lumínico de espacios emocionales. Anales de Edificación, 2(1), pp. 7-11.

De Luca, L., and Carboni, N., 2016. Towards a conceptual foundation for documenting tangible and intangible elements of a cultural object. Digital Applications in Archaeology and Cultural Heritage, 3(4), doi.org/10.1016/j.daach.2016.11.001.

Di Giulio, Maietti, F., Piaia, E., Medici, M., Ferreri, F., and Turillazzi, B., 2017. Integrated data capturing requirements for 3D semantic modelling of cultural heritage: the INCEPTION Protocol. The International Archives of Photogrammetry, Remote Sensing and Spatial Information Sciences, Vol. XLII2/W3, doi.org/10.5194/isprs-archives-XLII-2-W3-251-2017.

Di Luggo, A., and Catuogno, R., 2016. From point cloud to HBIM. Survey and modelling for the knowledge and management of architectural heritage. In T. Emper (ed.), $3 D$ Modelling and BIM, Applicazioni e posibili futuri sviluppi. Sapienza Università di Roma, Roma, pp. 58-64.

Di Luggo, A., and Scandurra, S., 2016. The knowledge of the architectural heritage in HBIM systems: from the discrete model to the parametric model. Disegnarecon 9(16), http://disegnarecon.univaq.it/ojs/index.php/disegnarecon/article/ view/148/117 (1 October 2018).

Fassi, F., Fregonese, L., Ackermann, S., and De Troia, V., 2013. Comparison between laser scanning and automated 3D modelling techniques to reconstruct complex and extensive cultural heritage areas. The International Archives of Photogrammetry, Remote Sensing and Spatial Information Sciences, 40-5/W1, pp. 73-80.
Guidi, G., Russo, M., and Angheleddu, D., 2014. 3D survey and virtual reconstruction of archaeological sites. Digital Applications in Archeology and Cultural Heritage, doi.org/10.1016/j.daach.2014.01.001.

Hsu, S.H., Chuang, M.C., and Chang, C.C., 2000. A semantic differential study of designers' and users' product from perception. International Journal of Industrial Ergonomics, 25(4), pp. 375-391, doi.org/10.1016/S0169-8141(99)00026-8.

Post Sennehed, Ch., Gard, G., Holmberg, S., Stigmar, K., Forsbrand, M., and Grahn, B., 2017. 'Blue flags', development of a short clinical questionnaire on work-related psychosocial risk factors - a validation study in primary care. $B M C$ Muskuloskeletal Disorders, 18(318), doi.org/10.1186/s12891017-1677-z.

Remondino, F., and Campana, S., (eds), 2014. 3D Recording and Modelling in Archaeology and Cultural Heritage. Theories and best practices. BAR International Series, Oxford.

Rinaudo, F., Bornaz, L., and Ardissone, P., 2007. 3D high accuracy survey and modelling for cultural heritage documentation and restoration. In Vast 2007-Future technologies to empower heritage professionals. Archaeolingua Hun, Broughton, UK, pp. 19-23. 